\title{
INTERVENCIÓN TEMPRANA EN NIÑOS CON ALTO RIESGO DE DESARROLLAR PARÁLISIS CEREBRAL; UNA REVISIÓN SISTEMÁTICA
}

\author{
EARLY INTERVENTION IN INFANTS AT HIGH-RISK OF DEVELOPING CEREBRAL \\ PALSY; A SYSTEMATIC REVIEW
}

\section{Daniela Wachholtz ${ }^{1}$ y Andrea Cortés ${ }^{2}$}

\begin{abstract}
Resumen
El objetivo fue determinar la evidencia existente acerca de la efectividad de intervenciones tempranas sobre el desarrollo motor de niños con alto riesgo de desarrollar una parálisis cerebral. Método: Se realizó una búsqueda literaria en bases de datos y revistas relevantes para el tema (CINAHL, The Cochrane Library, EMBASE, PEDro y PubMed). Los criterios de selección consideran a recién nacidos muy prematuros (<32 semanas de gestación) y/o con extremo bajo peso al nacer $(<1.500$ g), recién nacidos con encefalopatías neonatales y recién nacidos (prematuros o de término) que presentan resonancia magnética anormal y/o ultrasonido craneal anormal y/o la evaluación de movimientos generales alterada. Las calidades metodológicas de los estudios se evalúan con la escala PEDro y el nivel de evidencia con la clasificación del centro basado en la Evidencia de Oxford. Resultados: Se analizaron 9 estudios, comprendiendo 687 lactantes menores de 18 meses, quienes reflejan una gran variedad respecto al tipo y frecuencia de intervenciones y las herramientas de evaluación utilizadas. Los componentes terapéuticos asociados a efectos beneficiosos son: la participación de los padres en las intervenciones y la estimulación a la producción del movimiento por parte del bebé durante las actividades de la vida diaria. Discusión: Se evidencia que programas de intervención temprana que incluyen a cuidadores para promover el desarrollo motor y cognitivo, parecen tener más efectos beneficiosos en el largo plazo. Se requiere más investigación sobre este tema, y mejoras metodológicas para resultados más consistentes y así establecer el nivel de eficacia de los programas de intervención temprana.
\end{abstract}

\section{Palabras clave:}

parálisis cerebral, intervención temprana, niños con alto riesgo.

\begin{abstract}
The goal was to determine the evidence on the effectiveness of early interventions on motor development in children at high risk of developing cerebral palsy. Method: A literature search was conducted in databases and journals relevant to the topic (CINAHL, The Cochrane Library, EMBASE, PEDro and PubMed). Selection criteria considered very preterm infants ( $<32$ weeks' gestation) and/or with extremely low birth weight $(<1,500 \mathrm{~g}$ ), infants with neonatal encephalopathy and newborns (preterm or term) that have abnormal MRI and/ or abnormal cranial ultrasound and / or evaluation of altered general movements. Methodological quality of the studies was assessed with the PEDro scale and level of evidence with the classification based center for Evidence of Oxford scale. Results: 9 studies were analyzed, comprising 687 infants under 18 months, which reflect a variety regarding the type and frequency of interventions and evaluation tools used. The therapeutic benefits associated components are: the involvement of parents in interventions and stimulation to production of movement by the baby during activities of daily living. Discussion: It is evident, that early intervention programs which include caregivers to promote motor and cognitive development seem to have more beneficial effects in the long run. More research on this subject, and methodological improvements for more consistent results is required, to establish the level of effectiveness of early intervention programs.
\end{abstract}

\section{Keywords:}

cerebral palsy, early intervention, infants at high-risk.

Fecha de recepción: 26/08/2016.

Fecha de aceptación: 24/10/2016.

1 Terapeuta Ocupacional, DPS, MA. Teléfono +56-2-2618-2058. Email: dwachholtz@uandes.cl.Académico Universidad de Los Andes.

2 Terapeuta Ocupacional, MSc. Teléfono +56-2-29693459. Email: andre.cortesc@gmail.com. 


\section{INTRODUCCIÓN}

La parálisis cerebral (PC) es la causa más común de trastorno motor en la infancia y se caracteriza por una alteración en el movimiento y la postura, que compromete el desarrollo funcional del niño (Mclntyre et al., 2013; Jones et al., 2007). A nivel mundial, esta condición afecta alrededor de 2 a 2,5 por 1.000 nacidos vivos (Ashwal \& Russman, 2004; Herskind et al., 2014), pero esta cifra podría ser presumiblemente mayor en países menos desarrollados (Donald et al., 2014). A pesar de los avances en la comprensión conceptual de los factores de riesgo, no ha habido una disminución significativa en la incidencia y prevalencia de la PC a través del tiempo (Beaino et al., 2010; McIntyre et al., 2013). Un importante contribuyente a esto son los avances en la atención neonatal (Aisen et al., 2011) que han dado lugar a un aumento en el número de niños sobrevivientes nacidos muy prematuros $(<32$ semanas de gestación) o con extremo bajo peso (EBPN) $(<1.500 \mathrm{~g})$ y que presentan mayor riesgo de desarrollar una PC (Beaino et al., 2010; Odding et al., 2006).

La evidencia desde la neurociencia sugiere que intervenciones repetitivas con un alto nivel de intensidad y dirigidas hacia tareas específicas debiesen comenzar tan pronto como sea posible, cuando el sistema nervioso tiene una mayor capacidad para la reorganización y adaptación hacia las lesiones (Mclntyre, Morgan, et al., 2011). Sin embargo, la práctica más común en niños pequeños con sospecha de disfunción neurológica es "esperar y observar la evolución" antes de realizar un diagnóstico definitivo y comenzar con una intervención específica (McIntyre, Morgan, et al. 2011, pp. 120, y 121). En consecuencia, la mayoría de los niños con PC son diagnosticados entre los 18 a 24 meses de vida (Hielkema et al. 2010; Hadders-Algra, 2014; McIntyre, Morgan, et al., 2011), cuando el período de mayor plasticidad del cerebro comienza a decrecer (Graaf-Peters \& Hadders-Algra 2006). A raíz de esto se plantea una pregunta importante: $¿$ Los niños con alto riesgo de desarrollar PC lograrían un desarrollo funcional mejor si fuesen identificados precozmente y recibieran intervención temprana específica?

\section{ANTECEDENTES}

La importancia de esta revisión radica en que los estudios que se han efectuado previamente para determinar la efectividad de los programas de intervención temprana (PITs) han sido realizados en bebés prematuros (principalmente moderados y/o tardíos) y en lactantes que presentan algún nivel de retraso motor, pero no necesariamente en aquellos niños con mayor riesgo de desarrollar PC (Herskind et al., 2014). Por otra parte, los resultados de estos estudios han demostrado que los beneficios sobre el desarrollo motor no son claros (Spittle et al., 2012). Por lo tanto, sigue siendo un desafío importante identificar cuáles son los PITs más efectivos, seguros y prácticos para esta población, basados no solo en la experiencia clínica, sino también en la evidencia científica (Holt \& Mikati, 2011).

\section{MARCO CONCEPTUAL}

\section{La plasticidad neuronal y sus implicancias para la intervención temprana}

Décadas de investigación en neurobiología han demostrado que el desarrollo del sistema nervioso está continuamente influenciado no solo por la genética, sino también por el entorno (Knudsen 2004), Mientras los genes establecen el período específico en que se construyen los circuitos neuronales, los estímulos ambientales determinan el tipo y cantidad de conexiones sinápticas que se producen (Bischof, 2007). Por otra parte, aun cuando las investigaciones muestran que el desarrollo del cerebro es un proceso continuo que comienza in utero y se prolonga hasta la edad adulta, la mayoría de estas conexiones neuronales se establecen durante los primeros años de vida (Hensch, 2004), más concretamente alrededor de los 15 meses, cuando el proceso neurobiológico del desarrollo del cerebro se caracteriza por una marcada tendencia a la mielinización (Graaf-Peters \& Hadders-Algra, 2006).

Algunos estudios efectuados con animales después de sufrir una lesión cerebral temprana han encontrado que los períodos de mayor crecimiento dendrítico y de formación de sinapsis activas ofrecen mejores oportunidades para lograr resultados funcionales que las fases posteriores (Nimchinsky et al, 2002; Kolb et al., 2001). La extrapolación de estos estudios podría sugerir que el período entre las 28 semanas de gestación y 15 meses de edad postnatal podría ofrecer mayores posibilidades de intervenciones efectivas (Graaf-Peters \& HaddersAlgra, 2006).

Otros estudios han sugerido que existen dos tipos de experiencias ambientales relacionadas con mejores 
resultados: ser criado en un ambiente enriquecido, y recibir estimulación táctil durante los primeros años de vida (Kolb et al. 2001; Kolb et al. 2011). Los animales con una lesión cerebral temprana que se han criado en un ambiente enriquecido, incluyendo un espacio más grande y juguetes atractivos, muestran mejores resultados motores y cognitivos en comparación con los animales lesionados que son criados en un entorno de laboratorio ("no enriquecido") convencional (Kolb et al., 2001; Kolb et al., 2011). En estos casos, la recuperación de la funcionalidad está vinculada a aumentos en la longitud dendrítica, espesor cortical y el peso del cerebro. Complementariamente, otros estudios sugieren que el efecto del entorno enriquecido puede aumentarse por estimulación táctil temprana y cuidado materno (Hadders-Algra, 2014). En este sentido, se ha encontrado que la estimulación táctil (en forma de lamer y aseo personal de las crías lesionadas) está relacionada con un mejor rendimiento del motor y una mayor densidad de la espina dendrítica, lo que podría ser influenciado por el aumento de los niveles de factores neurotróficos (Kolb et al., 2013).

\section{Identificación temprana de niños con alto riesgo de desarrollar parálisis cerebral}

Debido a que existen múltiples factores de riesgo asociados a la PC, es muy difícil establecer con exactitud cuáles son los niños que están en mayor riesgo de desarrollar esta condición. Sin embargo, para los fines de esta revisión se han establecido tres grupos El primer grupo está constituido por los recién nacidos con encefalopatía neonatal (EN). La tasa de riesgo de desarrollar PC en estos bebés fluctúa entre 100-125/1000 sobrevivientes neonatales, por lo tanto representan el grupo de mayor riesgo de sufrir esta condición entre todos los niños (McIntyre, Morgan, et al., 2011). El segundo grupo abarca a los recién nacidos prematuros y de bajo peso al nacer, cuyo riesgo de desarrollar PC varía entre 3-80/1000 sobrevivientes neonatales (Mclntyre, Morgan, et al 2011; Jones et al., 2007). En general, la prevalencia de la PC disminuye a medida que aumenta la edad gestacional (Himpens et al., 2008; Mclntyre, Morgan, et al., 2011). Como consecuencia, las tasas de PC varían entre un $14,6 \%$ en los recién nacidos extremadamente prematuros ( $<28$ semanas de gestación), un $6,2 \%$ en los bebés nacidos entre las 28 y 31 semanas de gestación, hasta el $0,7 \%$ en recién nacidos prematuros moderada (32-<37 semanas de gestación) (Himpens et al., 2008). Por último, el tercer grupo de niños en potencial riesgo está compuesto por los recién nacidos de término y sin ningún signo de anormalidad que reciben atención de rutina básica. Para este subgrupo de niños el riesgo de PC es 1/1000 sobrevivientes neonatales $y$, por ende, representan el grupo de menor riesgo (Mclntyre, Morgan, et al., 2011).

La identificación temprana de los bebés con alto riesgo de PC es crucial para iniciar los programas específicos de intervención temprana y facilitar el desarrollo funcional en la infancia (Herskind et al., 2014). Además, el periodo que comprende desde la sospecha de PC hasta que se proporciona el diagnóstico final a los padres y puede ser muy estresante y debe reducirse cuando es posible (Baird et al., 2000). De acuerdo con esto, un diagnóstico precoz del niño complementado con apoyo de salud mental preventiva para los padres podría ser no solo beneficioso para reducir sus niveles de estrés y ansiedad, sino también para introducir intervenciones que maximicen los beneficios otorgados por períodos de mayor plasticidad neuronal (McIntyre, Morgan, et al., 2011). A pesar de lo anterior, el diagnóstico precoz de PC es todavía muy difícil debido a una serie de factores. En primer lugar, no existen marcadores biológicos o pruebas definitivas que aseguren su reconocimiento temprano (Mclntyre, Morgan, et al., 2011). En segundo lugar, los cambios en el desarrollo continuo del cerebro y los síntomas generalizados y no específicos presentados por los niños pequeños también obstaculizan su predicción en la edad temprana. Y, en tercer lugar, los médicos deben descartar otras patologías que presentan características similares a la PC, con el fin de seleccionar las estrategias de intervención más adecuados para cada niño (Bosanquet et al., 2013). A pesar de todos estos factores, la investigación sugiere que la predicción de PC se puede mejorar significativamente mediante el uso de las evaluaciones de neuroimagen y neuromotora general (Skiold et al., 2013).

Las técnicas de neuroimagen, en particular, el ultrasonido craneal (USC) y la imagen por resonancia magnética (IRM), son esenciales para la determinación de la patogénesis de la PC durante el proceso de diagnóstico (Krägeloh-Mann \& Horber, 2007). El USC es un procedimiento de bajo costo que se aplica con mayor frecuencia en los bebés prematuros durante su estadía en la unidad de cuidados intensivos neonatales para identificar los cambios estructurales del cerebro en desarrollo (Mclntyre, Morgan, et al., 2011). De acuerdo con el metaanálisis realizado por Bosanquet y colaboradores (2013), que incluyó a más de 2.400 recién nacidos prematuros, 
el poder de la USC para predecir la PC durante la etapa neonatal arroja un $74 \%$ de sensibilidad y un $92 \%$ de especificidad. Por otra parte, la IRM es la técnica "gold standard" para determinar alteraciones neurológicas en los recién nacidos, especialmente en aquellos con edad de término (Hadders-Algra, 2014; De Vries et al., 2011). Esta técnica puede pronosticar la PC con una sensibilidad entre el $86-100 \%$ y una especificidad que va desde 89 al 97\%, lo que refleja un alto nivel predictivo (Bosanquet et al., 2013). A pesar de esto, es importante señalar que aproximadamente el $12 \%$ de los niños con PC pueden mostrar IRM normales y por lo tanto esta técnica debe complementarse con evaluaciones adicionales con el fin de lograr resultados más fiables (Bax et al., 2005; Krägeloh-Mann \& Horber, 2007). Una de las evaluaciones complementarias más común para bebés de alto riesgo es General Movement Assessment (GMA). Como expresa Herskind, (2014, pp. 32), "esta técnica consiste en una observación de la calidad de los patrones de movimiento espontáneo, donde los movimientos generales anormales indican un alto riesgo de trastornos del desarrollo como una PC". GMA ha demostrado ser una de las herramientas más potentes y eficaces para predecir la PC, evidenciando altos índices de especificidad (91\%) y sensibilidad (98\%) (Burger \& Louw, 2009; Bosanquet et al., 2013). Además, diversos autores recomiendan la combinación de los resultados de GMA y de IRM en niños a edad de término, ya que así aumenta la especificidad de predicción al 100\% (Skiöld et al., 2013).

\section{Programas de intervención temprana}

La intervención temprana (IT) puede ser definida como "servicios multidisciplinarios proporcionados a los niños desde el nacimiento hasta los 5 años de edad, para promover la salud y el bienestar infantil, mejorar las competencias emergentes, minimizar los retrasos en el desarrollo, remediar discapacidades existentes o emergentes, prevenir el deterioro funcional, fomentar la paternidad responsable y promover un adecuado funcionamiento familiar" (Zigler, 2000, prólogo, pp. xvii-xviii). Además, las intervenciones tempranas del desarrollo pueden incluir servicios de diversas disciplinas tales como psicología, terapia ocupacional, educación y kinesiología (BlauwHospers \& Hadders-Algra, 2005). Es importante precisar que la definición del término "temprana" ha sido motivo de controversia, ya que puede ser entendido de dos formas: temprana en la vida o temprana en la expresión de una disfunción, siendo ambas interpretaciones asociadas con aspectos positivos y negativos para la intervención (Blauw-Hospers \& Hadders-Algra, 2005). El mayor beneficio de iniciar las intervenciones terapéuticas temprano en la vida es que el cerebro presenta mayores niveles de plasticidad que pueden maximizar las oportunidades para lograr mejores resultados motores (Hadders-Algra 2001). Por otra parte, sabemos que los efectos secundarios que resultan de una lesión cerebral pueden producir períodos prolongados de dolor y estrés para el bebé (Holt \& Mikati, 2011). Se sabe también que el estrés durante la vida temprana tiene consecuencias de por vida, ya que puede inducir cambios permanentes en el cerebro (Holt \& Mikati, 2011) y déficits en los sistemas monoaminérgicos y dopaminérgicos (Weinstock, 2008; Van Den Bergh et al., 2005). Por lo tanto, es deseable que la intervención para los niños con una lesión cerebral comience tan pronto como sea posible, para reducir los niveles de estrés y dolor (Hadders-Algra, 2014). Contrariamente, las intervenciones que comienzan temprano en la vida pueden también estar asociadas con potenciales desventajas. Por un lado, el tipo de problemas que el niño desarrollará más adelante no está del todo claro, lo que hace más difícil establecer metas específicas de tratamiento (Badr et al., 2006). Por otro lado, es probable que algunos niños con sospecha de riesgo no presenten un deterioro motor más tarde, por lo que la intervención puede no ser necesaria para ellos (Blauw-Hospers \& Hadders-Algra, 2005). En los últimos años, los PITs han demostrado efectos positivos sobre las habilidades cognitivas y sociales de niños en situación de pobreza y vulnerabilidad (Zigler, 2000). Poco a poco, la evidencia ha surgido también en relación a los efectos de las intervenciones tempranas en los niños con riesgo de deterioro neuromotor, tales como los nacidos prematuramente. Estos estudios han indicado que la IT se asocia con mejoras en el desarrollo cognitivo durante la infancia y la edad preescolar, pero, la evidencia de efectos positivos sobre la función motora no es determinante (Spittle et al., 2012; Hadders-Algra, 2011).

\section{Metodología}

Criterios para la búsqueda literaria: Esta revisión sistemática incluye ensayos clínicos que utilicen una asignación aleatoria o cuasi aleatoria y que cumplan con los criterios para tipo de intervenciones y tipos de participantes, ambos descritos a continuación. Para los efectos de esta revisión, los niños de alto riesgo se definen como aquellos que cumplen uno o más de los siguientes cri- 
terios: (1) muy prematuros ( $<32$ semanas de gestación) y/o con EBPN ( $<1.500$ g), (2) recién nacidos con EN, (3) lactantes (prematuros o nacidos a término) con IRM y/o USC claramente anormal y/o GMA alterado. Además, los niños deben tener una edad igual o menor a 18 meses al inicio del programa. Los estudios excluidos de esta revisión son aquellos basados en población de recién nacidos prematuros con menor riesgo ( $>32$ semanas de gestación y/o >1.500 g), niños con retraso en el desarrollo debido a otras condiciones (tales como el síndrome de Down) o niños en otras situaciones de vulnerabilidad, pero sin riesgo de parálisis cerebral. Por otra parte, esta revisión incluye PITs cuyo objetivo contemple mejorar el desarrollo motor infantil. Estos programas deben comenzar durante los primeros 18 meses del niño, pudiendo ser efectuados en un hospital, centro comunitario o en el hogar. Además, la intervención debe ser proporcionada por un profesional del área de la salud. Los estudios restringidos solo a intervenciones quirúrgicas, ortopédicas y farmacéuticas o destinadas exclusivamente a mejorar las habilidades cognitivas de los niños, fueron excluidos de esta revisión.

Estrategia de búsqueda: La búsqueda bibliográfica se efectuó en los meses de julio y agosto de 2015 a través de las siguientes bases de datos: CINAHL, The Cochrane
Library, EMBASE, PEDro y PubMed. Esta búsqueda fue complementada con la revisión de sitios web de PC internacionalmente reconocidos, tales como (1) la Academia Americana de Parálisis Cerebral y Medicina del Desarrollo (www.aaPCdm.org), (2) la Alianza Parálisis Cerebral (www.cerebralpalsy.org.au), (3) CanChild (www. canchild.ca) y (4) NeuroDevNet (www.neurodevnet.ca), y mediante búsquedas manuales en las siguientes revistas académicas: (1) Developmental Medicine and Child Neurology y (2) Pediatric Physical Therapy. El detalle completo de la estrategia de búsqueda puede ser proporcionado por los autores según sea requerido.

Criterios de inclusión y de exclusión: Los estudios incluidos en esta revisión debían cumplir con los criterios anteriormente mencionados (tipos de estudios, tipos de participantes y tipos de intervenciones). Además, los artículos debían estar completamente disponibles, en idioma inglés y ser publicados desde 1990. Los artículos fueron excluidos si no se encontraban completamente disponibles, en un idioma distinto al inglés, publicados antes de 1990 o si no cumplían los criterios previamente mencionados.

Selección de los estudios: Posterior al proceso de búsqueda, un total de 9 artículos fueron seleccionados para esta revisión, como se indica en el diagrama de flujo:

Figura 1:

Diagrama de flujo de artículos seleccionados.

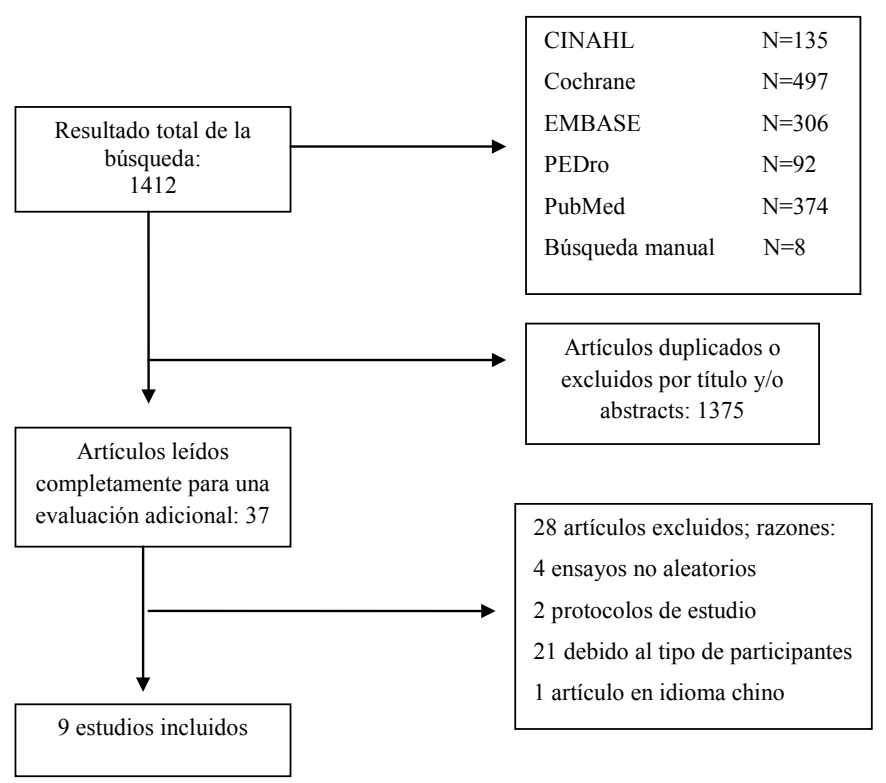




\section{Resultados}

Los nueve estudios que satisfacen los criterios de inclusión son analizados en esta sección. La Tabla 1 describe las características generales de cada estudio, incluyendo el año de publicación, tamaño de la muestra, grupo de estudio, tipo de diseño, nivel de evidencia y calidad metodológica. A partir de esto se observa que la suma total de niños bajo análisis es 687; sin embargo, el número de participantes en cada ensayo varía entre 23 a 176. Además, en 4 estudios la muestra consiste en bebés prematuros nacidos en promedio $<30$ semanas de edad gestacional (EG) y con EBPN, mientras que en los otros 5 estudios los participantes son lactantes (prematuros o no) con lesiones cerebrales expresadas por ultrasonido craneal claramente anormal, resonancia magnética atípica o GMA alterado. Es importante destacar que todos los estudios corresponden a ECAs y su calidad metodológica fue evaluada de acuerdo a la escala PEDro (Maher et al., 2003; Verhagen et al.,1998). Como resultado, cuatro estudios obtuvieron una puntuación igual o mayor a 6 (alta calidad metodológica), mientras que cinco obtuvieron una puntuación de 5 (calidad metodológica media). Además, el nivel de evidencia de los estudios se determinó a través de la clasificación de Oxford, con base en el Centro de Medicina Basada en la Evidencia (CEBM) (Fernández Rego et al., 2012). De acuerdo con esta escala, el total de los estudios obtienen grado $1 \mathrm{~b}$, lo que significa buen nivel de calidad.

Tabla 1:

Características metodológicas de los estudios.

\begin{tabular}{|c|c|c|c|c|c|c|c|c|}
\hline \multirow{2}{*}{ Estudios } & \multirow{2}{*}{ Año } & \multicolumn{3}{|c|}{ Tamaño de la muestra } & \multirow{2}{*}{ Grupo de estudio } & \multirow{2}{*}{ Diseño } & \multirow{2}{*}{$\begin{array}{l}\text { Nivel de evi- } \\
\text { dencia }\end{array}$} & \multirow{2}{*}{$\begin{array}{l}\text { Escala } \\
\text { PEDro }\end{array}$} \\
\hline & & $\mathrm{N}$ & $n-E$ & $n-C$ & & & & \\
\hline Badr et al. & 2006 & 62 & 32 & 30 & NAR con daño SNC & ECA & $1 b$ & $5 / 10$ \\
\hline $\begin{array}{c}\text { Blauw-Hospers } \\
\text { et al. }\end{array}$ & 2011 & 46 & 21 & 25 & NAR con GMA-A & ECA & $1 b$ & $6 / 10$ \\
\hline Cameron et al. & 2005 & 72 & 34 & 38 & $\mathrm{P}<32$ SG y/o $<1500 \mathrm{~g}$ & ECA & $1 b$ & $5 / 10$ \\
\hline Hielkema et al. & 2011 & 46 & 21 & 25 & NAR con GMA-A & ECA & $1 b$ & $6 / 10$ \\
\hline Koldewijn et al. & 2009 & 176 & 86 & 90 & $\mathrm{P}<32 \mathrm{SG} y / \mathrm{o}<1500 \mathrm{~g}$ & ECA & $1 b$ & $7 / 10$ \\
\hline Nelson et al. & 2001 & 37 & 21 & 16 & $\begin{array}{l}\mathrm{P}<26 \text { SG y/o }<1500 \mathrm{~g}, \mathrm{o} \\
\mathrm{P}<32 \text { SG con daño } \mathrm{SNC}\end{array}$ & ECA & $1 b$ & $5 / 10$ \\
\hline Ohgi et al. & 2004 & 23 & 12 & 11 & NAR con daño SNC & ECA & $1 b$ & $5 / 10$ \\
\hline Spittle et al. & 2010 & 120 & 61 & 59 & $\mathrm{P}<30 \mathrm{SG}$ & ECA & $1 \mathrm{~b}$ & $6 / 10$ \\
\hline Weindling et al. & 1996 & 105 & 51 & 54 & NAR con daño SNC & ECA & $1 b$ & $5 / 10$ \\
\hline
\end{tabular}

$\mathrm{N}$ : Número total de niños; n-E: $\mathrm{N}^{\circ}$ de niños en el grupo experimental; $\mathrm{n}-\mathrm{C}: \mathrm{N}^{\circ}$ de niños en el grupo control; NAR: Niños de Alto Riesgo; SNC: Sistema Nervioso Central; GMA-A: General Movement Assessment-Abnormal (evaluación de movimientos generales alterada); P: Bebés Prematuros; SG: Semanas de Gestación, ECA: Ensayo Clínico Aleatorizado; *Los daños en el SNC refieren un examen de ultrasonido y/o resonancia magnética alterados. 
A continuación se resumen las características generales de los programas de intervención realizados en cada estudio, junto a sus respectivos resultados. La Tabla 2 describe el tipo de intervención aplicada, la edad de los niños al iniciar y finalizar el programa, la persona encargada de proporcionar la intervención y el lugar donde ésta se llevó a cabo. A partir de esta información es posible observar los diversos métodos de tratamiento aplicados en cada estudio. La mayoría de los programas incluyen procedimientos para reducir el estrés ambiental, como disminución de sonidos y luces, regulación del ciclo sueño-vigilia (especialmente el iniciado en la unidad de cuidados intensivos neonatales), técnicas para mejorar el rendimiento motor (siendo la técnica NDT una de las más utilizadas), estimulación multisensorial y una amplia gama de estrategias para fomentar una adecuada relación cuidador-niño. Notablemente, la mayoría de las intervenciones aplicadas después del alta se llevaron a cabo en el hogar de los participantes; sin embargo, el lugar de intervención pareciera no afectar la eficacia del tratamiento.

A pesar de la gran diversidad de estrategias de intervención aplicadas en los 9 ensayos clínicos, es posible observar diferencias consistentes entre los programas de intervención temprana orientados a niños prematuros que aún no han alcanzado la edad de término, en comparación a aquellos dirigidos a niños que ya la completaron. Las intervenciones antes de la edad de término se centran principalmente en imitar el ambiente intrauterino y en reducir el estrés (Nelson et al., 2001; Ohgi et al., 2004; Hadders-Algra, 2011), mientras que las intervenciones en niños con edad de término se orientan a la estimulación general del desarrollo infantil (Weindling et al., 1996; Hadders-Algra, 2011).

Tabla 2:

Características generales de los PIT.

\begin{tabular}{|c|c|c|c|c|c|}
\hline \multirow{2}{*}{ Estudios } & \multirow{2}{*}{ Tipo de intervención } & \multicolumn{2}{|c|}{ Edad de los participantes } & \multirow{2}{*}{$\begin{array}{c}\text { Persona a cargo de la inter- } \\
\text { vención }\end{array}$} & \multirow{2}{*}{ Lugar de intervención } \\
\hline & & al inicio & al final & & \\
\hline Badr et al. & CAMS/EP & $\mathrm{AH}-1 \mathrm{M}$ & $12 \mathrm{M} \mathrm{EC}$ & $\mathrm{E} / \mathrm{P}$ & Hogar \\
\hline $\begin{array}{c}\text { Blauw-Hospers } \\
\text { et al. }\end{array}$ & COPCA & $3 \mathrm{Ms}$ EC & $6 \mathrm{M} \mathrm{EC}$ & $\mathrm{FT} / \mathrm{P}$ & Hogar \\
\hline \multirow{2}{*}{ Cameron et al. } & NDP & \multirow{2}{*}{ Previo a $\mathrm{AH}$} & \multirow{2}{*}{$4 \mathrm{M} \mathrm{EC}$} & \multirow{2}{*}{ FT } & Hospital \\
\hline & EP & & & & Hogar \\
\hline Hielkema et al. & COPCA & $3 \mathrm{Ms}$ EC & $6 \mathrm{M} \mathrm{EC}$ & $\mathrm{FT} / \mathrm{P}$ & Hogar \\
\hline \multirow{2}{*}{ Koldewijn et al. } & IBAIP & \multirow{2}{*}{ Previo a $\mathrm{AH}$} & \multirow{2}{*}{$6 \mathrm{M} \mathrm{EC}$} & \multirow{2}{*}{$\mathrm{FT} / \mathrm{P}$} & Hospital \\
\hline & EP & & & & Hogar \\
\hline \multirow{2}{*}{ Nelson et al. } & $\begin{array}{l}\text { EMS } \\
\text { SRP }\end{array}$ & \multirow{2}{*}{33 Sems PC } & \multirow{2}{*}{$2 \mathrm{M} \mathrm{EC}$} & \multirow{2}{*}{$\mathrm{P} / \mathrm{O}$} & Hospital \\
\hline & EP & & & & Hogar \\
\hline \multirow{3}{*}{ Ohgi et al. } & NBAS & \multirow{3}{*}{ Previo a $\mathrm{AH}$} & \multirow{3}{*}{$6 \mathrm{M} \mathrm{EC}$} & \multirow{3}{*}{$\mathrm{FT} / \mathrm{P}$} & \multirow{2}{*}{ Hospital } \\
\hline & NDT & & & & \\
\hline & EP & & & & Hogar \\
\hline Spittle et al. & VIBeS Plus & 1-2 S EC & $11 \mathrm{M} \mathrm{EC}$ & $\mathrm{FT} / \mathrm{PS}$ & Hogar \\
\hline \multirow{2}{*}{ Weindling et al. } & NDT & \multirow{2}{*}{40 sems $\mathrm{P}-\mathrm{C}$} & \multirow{2}{*}{$12 M$} & \multirow{2}{*}{ FT } & \multirow{2}{*}{ Hogar } \\
\hline & EP & & & & \\
\hline
\end{tabular}


CAMS: Curriculum and Monitoring System (basado en estimulación sensorio motora y cognitiva), EP: Educación Parental, COPCA: Coping with and Caring for infants with special needs (basado en educación y entrenamientos a los padres), NDP Neonatal Developmental Program (basado en la técnica Bobath), IBAIP: Infant Behavioural Assessment and Intervention Programme (basado en promover las competencias autoregulatorias del niño y aumentar las habilidades de los cuidadores para interpretar y responder las señales de sus bebés), EMS: Estimulación multi-sensorial (auditiva, táctil, visual y vestibular),SRP: Stress Reduction Program, NBAS: Neonatal Behavioural Assessment Scale (basado en mejorar la interacción bebé-cuidador), NDT: NeurodevelopmentalTreatment (método Bobath), VIBeS PLUS: Victorian Infant Brain Study Plus (basado en educación parental y estimulación motora), AH: Alta Hospitalaria, M: Meses, EC: Edad Corregida, Sems: semanas, P-C: Post-concepción, FT: Fisioterapeuta, E: Enfermera, P: Padres, PS: Psicólogo y O: Otro (en este caso, el equipo de investigadores).

La Tabla 3 enumera los instrumentos de evaluación utilizados, las edades de los niños cuando éstos fueron aplicados, la frecuencia e intensidad de la intervención y un resumen de los resultados principales. En base a esto, es posible observar una gran variación en cuanto a los instrumentos de evaluación utilizados, siendo la escala Bayley de Desarrollo Infantil la más utilizada por los estudios bajo análisis. Respecto a la frecuencia e intensidad con que las intervenciones fueron aplicadas, existen variaciones que van desde dos veces al día a una vez al mes.

La última columna de la Tabla 3 muestra que sólo 1 estudio logró resultados estadísticamente significativos. Koldewijn y col. (2009) Ilevaron a cabo un ensayo aleatorio multicéntrico para evaluar la eficacia del "programa de evaluación e intervención conductual para infantes" (IBAIP, abreviación en inglés) en bebés prematuros nacidos con $<32$ semanas de gestación y/o $<1500$ gramos. El grupo de intervención $(\mathrm{N}=86)$ recibió una sesión antes del alta hospitalaria, la cual fue seguida por 6 a 8 visitas domiciliarias hasta los 6 meses de edad corregida, las cuales fueron llevadas a cabo por kinesiólogos pediátricos entrenados en la técnica IBAIP. En comparación, el grupo control $(n=90)$ recibió un seguimiento estándar que consistió principalmente en visitas regulares al pediatra. En caso de ser necesario, el médico podía derivar a los bebés del grupo control a sesiones de kinesiología, pero no con aquellos profesionales entrenados en la técnica IBAIP. La intervención tuvo como objetivo promover las competencias autoregulatorias del niño y aumentar las habilidades de los cuidadores para interpretar y responder las señales de sus bebés durante la ejecución de actividades de la vida diaria. A los 6 meses, la escala Bayley de Desarrollo Infantil mostró que el programa mejoró el componente cognitivo y motor de los participantes del grupo experimental. 
Tabla 3:

Características generales de los PIT y resultados.

\begin{tabular}{|c|c|c|c|}
\hline \multirow{2}{*}{ Estudios } & Instrumentos de evaluación & \multirow{2}{*}{ Frecuencia/Intensidad } & \multirow{2}{*}{ Resultados } \\
\hline & (desarrollo motor) & & \\
\hline $\begin{array}{l}\text { Badr et al., } \\
2006\end{array}$ & $\begin{array}{c}\text { Aplicación 6, } 12 \text { y } 18 \text { meses EC: } \\
\text {-BSID (PDI) }\end{array}$ & $\begin{array}{l}\text { Dos veces por semana el primer mes, luego una } \\
\text { vez por semana hasta los } 4 \text { meses de edad y final- } \\
\text { mente cada dos semanas hasta que el niño cumpla } \\
\qquad 12 \text { meses. }\end{array}$ & $\mathrm{E}=\mathrm{C}$ \\
\hline $\begin{array}{l}\text { Blaw-Hospers et } \\
\text { al., } 2011\end{array}$ & $\begin{array}{c}\text { Aplicación 3, } 6 \text { y } 18 \text { meses EC: } \\
\text {-AIMS } \\
\text {-IMP } \\
\text {-PEDI }\end{array}$ & Dos veces por semana, cada sesión dura 1 hora. & $\mathrm{E}=\mathrm{C}$ \\
\hline $\begin{array}{l}\text { Cameron et al., } \\
2005\end{array}$ & $\begin{array}{l}\text { Aplicación } 4 \text { meses EC: } \\
\text {-AIMS. }\end{array}$ & $\begin{array}{c}\text { Todos los días, cada sesión dura } 10 \text { minutos. Pos- } \\
\text { terior al alta hospitalaria, los padres continúan la } \\
\text { intervención en casa. }\end{array}$ & $\mathrm{E}=\mathrm{C}$ \\
\hline Hielkema, 2011 & $\begin{array}{c}\text { Aplicación 3, 4, } 5 \text {,6 y } 18 \text { meses CA: } \\
\text {-Infant Motor Profile (IMP) }\end{array}$ & $\begin{array}{l}\text { Dos veces por semana. Sin información respecto a } \\
\text { la duración de las sesiones. }\end{array}$ & $\mathrm{E}=\mathrm{C}$ \\
\hline $\begin{array}{l}\text { Koldewijn et al., } \\
\qquad 2009\end{array}$ & $\begin{array}{c}\text { Aplicación } 6 \text { meses EC: } \\
\text {-BSID II (PDI) } \\
\text {-IBA }\end{array}$ & $\begin{array}{l}\text { Una sesión previa al alta hospitalaria. Posterior- } \\
\text { mente, 6-8 sesiones en el hogar con una duración } \\
\text { de una hora. }\end{array}$ & $\mathrm{E}>\mathrm{C}$ \\
\hline $\begin{array}{l}\text { Nelson et al., } \\
2001\end{array}$ & $\begin{array}{l}\text { Aplicación } 2 \text { meses EC: } \\
\text {-BSID (PDI) }\end{array}$ & $\begin{array}{l}\text { Sesión de } 15 \text { min., } 2 \text { veces al día, } 5 \text { días por sema- } \\
\text { na hasta el alta hospitalaria. En el hogar, la inter- } \\
\text { vención es continuada } 2 \text { veces al día por la madre. }\end{array}$ & $\mathrm{E}=\mathrm{C}$ \\
\hline $\begin{array}{l}\text { Ohgi et al., } \\
2004\end{array}$ & $\begin{array}{l}\text { Aplicación } 6 \text { meses EC: } \\
\text {-BSID (PDI) }\end{array}$ & $\begin{array}{l}\text { Previo al alta hospitalaria: 3-4 sesiones de } 30 \text { min. } \\
\text { Posteriormente, una sesión por semana. Cada } \\
\text { sesión dura 40-60 minutos. }\end{array}$ & $\mathrm{E}=\mathrm{C}$ \\
\hline $\begin{array}{l}\text { Spittle et al., } \\
2010\end{array}$ & $\begin{array}{l}\text { Aplicación } 24 \text { meses EC: } \\
\text {-BSID III (PDI) }\end{array}$ & $\begin{array}{c}\text { Durante el primer año de vida se realizan } 9 \text { se- } \\
\text { siones en el hogar, con una duración de } 1.5 \text { a } 2.0 \\
\text { horas. }\end{array}$ & $\mathrm{E}=\mathrm{C}$ \\
\hline $\begin{array}{l}\text { Weindling., } \\
1996\end{array}$ & $\begin{array}{c}\text { Aplicación } 12 \text { y } 30 \text { meses: } \\
\text { Motor Assessment of Infants } \\
\text { GRIFFITHS developmental scale }\end{array}$ & $\begin{array}{c}\text { Una sesión semanal hasta los } 6 \text { meses de edad, } \\
\text { luego cada } 15 \text { días hasta los } 9 \text { meses y finalmente } \\
\text { una vez al mes hasta los } 12 \text { meses. }\end{array}$ & $\mathrm{E}=\mathrm{C}$ \\
\hline
\end{tabular}

EC: Edad Corregida, BSID (PDI): Bayley Scale of Infant Development (Physical Development Index), AIMS: Alberta Infant Motor Scale, IMP: Infant Motor Profile, PEDI: Pediatric Evaluation of Disability Inventory, IBA: Infant Behavioural Assessment, NDS: Neurodevelopmental Scale, NSDMA: Neurological Sensory Motor Developmental Assessment y E=C: Grupo experimental y control, sin diferencias significativas en sus resultados al final de la intervención $\mathrm{E}>\mathrm{C}$ : Resultados favorables para el grupo experimental. 


\section{DISCUSIÓN Y CONCLUSIONES}

Todos los PITs analizados evidencian progresos en el desarrollo motor, aun cuando los avances observados no son estadísticamente significativos. Sin embargo, estos resultados deben ser tomados con cautela, ya que la heterogeneidad presentada en los estudios (grandes variaciones en términos del tamaño muestral, tipo de intervención proporcionada instrumento de evaluación utilizado, entre otros) dificulta la generalización de estas conclusiones e impide la realización de un meta-análisis. A partir de los resultados de esta revisión se abordan los aspectos más relevantes a tener en consideración durante la práctica clínica y futuras investigaciones.

\section{Implicancias para la práctica clínica}

La participación activa de los padres/cuidadores durante la intervención ha sido señalada en diversas ocasiones como un componente terapéutico fundamental para alcanzar mejores resultados en el desarrollo motor infantil (Blauw-Hospers et al., 2011; Dirks \& HaddersAlgra, 2011; Benzies et al., 2013). En términos generales, esta estrategia busca favorecer una mejor comprensión y respuesta de los padres hacia las necesidades manifestadas por sus hijos e instruirlos en diversas técnicas para estimular el desempeño motor del niño, especialmente durante las actividades de la vida diaria (Hielkema et al., 2011). Se ha indicado también que esto ayudaría a reducir los niveles de ansiedad y estrés de los padres, aumentar su sentido de autoeficacia (Ohgi et al., 2004; Spittle et al., 2010) y mejorar la adherencia al tratamiento (Hielkema et al., 2011). Sin embargo, la forma en que los padres son integrados a la intervención varía en todos los estudios bajo análisis. En algunos programas, la educación parental se centra principalmente en la entrega de información respecto al crecimiento y desarrollo infantil con espacios para aclarar dudas y temores de los padres, en otros incluye demostraciones por parte del profesional tratante, acerca de cómo promover posturas y movimientos en el niño, y finalmente, hay casos en que implica la práctica directa de los padres en el manejo postural del niño, con espacios de tiempo para experienciar otras técnicas de abordaje y recibir sugerencias del profesional a cargo de la intervención. La diversidad con que los padres/ cuidadores son integrados al tratamiento aumenta la heterogeneidad de las intervenciones, por lo que debie- se ser descrita con mayor profundidad en los protocolos de intervención.

Fomentar la producción de la propia conducta motora a través de una variedad de actividades funcionales y permitir que el niño juegue un papel activo en el tratamiento mediante el aumento de su motivación intrínseca, es otro componente de la intervención que ha sido asociado con un mejor resultado en el desarrollo motor (Blauw-Hospers et al., 2011). De acuerdo con esta idea, Herskind y colaboradores (2014) han sugerido que el aprendizaje motor se produce cuando el niño explora activamente el medio ambiente y encuentra soluciones a las demandas de la nueva tarea que se le presenta. Por lo tanto, los programas de intervención temprana no deben implicar solo la estimulación pasiva del niño, sino que requieren que éste desempeñe un papel activo. De esta forma, el terapeuta debe ser capaz de desafiar la conducta motora del menor con un nivel de exigencia adecuadamente alto para mantener la atención y motivación del niño durante la ejecución de las actividades y a la vez, evitar niveles de dificultad excesivos que puedan provocar su frustración y abandono de la tarea (Hielkema et al., 2011; Herskind et al., 2014).

Uno de los problemas observados en esta revisión se relaciona con los instrumentos de evaluación utilizados para medir los resultados de la intervención. En general, todos ellos muestran un bajo nivel de sensibilidad para detectar pequeños cambios en el rendimiento motor, aun cuando estos cambios pueden influir de manera significativa en las capacidades funcionales del niño (Siebes et al., 2002; Blouw-Hospers et al., 2005). Por otra parte, la mayoría de estos instrumentos miden cambios cuantitativos sobre la función motora, pero no siempre toman en cuenta las mejoras en la calidad de los movimientos o cambios en las habilidades funcionales (Ketelaar et al., 1998). Esto es de vital importancia para la interpretación de los resultados, ya que en muchas ocasiones la intervención se centra en la optimización de la calidad de la postura y el movimiento, con el objetivo de mejorar la funcionalidad del niño (Fernández Rego et al., 2012). Por otra parte, a pesar de que el actual modelo de la CIF resalta la importancia de los factores contextuales en el desarrollo del niño (Simeonsson et al., 2003), muy pocas escalas toman en cuenta la participación del niño en actividades acordes a su edad (como el juego) para determinar el impacto de la intervención. 


\section{Implicancias para futuras investigaciones}

A nivel general, se ha observado que muchos ECAs carecen de rigurosidad para determinar quiénes son los infantes con alto riesgo de desarrollar PC y en muchos casos la selección de participantes se basa exclusivamente en la prematuridad como un factor determinante para el desarrollo de PC (Herskind et al., 2014). Sin embargo, y como se ha expuesto previamente, a pesar de que los recién nacidos prematuros tienen un mayor riesgo de desarrollar PC en comparación a los niños "sanos" nacidos de término, la mayoría de ellos mostrará un desarrollo normal (Himpens et al., 2008). Por lo tanto, se requiere un proceso de identificación más riguroso de las poblaciones de alto riesgo con el fin de incluir a los recién nacidos que más se beneficiarían de los programas $y$, por ende, contribuir a reforzar la evidencia a favor de la intervención temprana en niños con PC (Spittle et al., 2010). Si esto no se realiza, la obtención de efectos significativos se dificulta pues el tratamiento aplicado a niños que no necesariamente requieren de la intervención puede disminuir el efecto terapéutico de los niños que sí la necesitan (Herskind et al., 2014).

Otro problema persistente en la investigación clínica sobre parálisis cerebral es la dificultad para reclutar un número adecuado de participantes (Siebes et al., 2002). Por desgracia, un tamaño reducido de la muestra puede resultar no solo en la disminución de la significancia estadística del estudio, sino que también dificulta su generalización en otros contextos (Majnemer, 1998; Blauw-Hospers \& Hadders-Algra, 2005). Un buen ejemplo de esto es el ensayo clínico realizado por Ohgi y colaboradores en 2004, en el que el tamaño muestral se estimó originalmente en 58 niños, pero que debido a dificultades en el proceso de reclutamiento solo incluyó a 23 participantes. Como resultado, el poder estadístico del estudio se redujo a un $40 \%$ aproximadamente. Frente a esto, la realización de ECAs multicéntricos se presenta como una alternativa promisoria para afrontar los desafíos metodológicos que conlleva un tamaño muestral reducido. Este fue el caso del estudio realizado por Koldewijn y colaboradores en el año 2009 y que presenta el número de participantes más grande de esta revisión.

La falta de un grupo control "verdadero" es un aspecto que también debe ser considerado. A medida que los PITs se han convertido en una intervención esencial para los niños con riesgo de compromiso motor, el reclutamiento de los participantes para un grupo control que no va a recibir tratamiento se considera poco ético (Siebes et al., 2002). Por ende, una cierta dosis de terapia "estándar" para estos niños es necesaria, lo que podría debilitar los resultados de la intervención bajo análisis. Por ejemplo, el grupo control en los estudios de Nelson (2001) y Blaw-Hospers (2011) recibió sesiones de "terapia estándar" que pudo haber influido en la función motora de estos niños. Por eso, podría conjeturarse que los resultados obtenidos por estos estudios solo reflejan el valor adicional de la intervención temprana y, por ende, diferencias significativas entre los grupos control y experimental son difíciles de lograr.

Es bien sabido que los niños con PC, especialmente aquellos con mayores niveles de compromiso, requieren de mayor tiempo y apoyo para lograr cambios en su desempeño motor (Hur, 1995). Por lo tanto, los efectos de los PITs no solo debiesen ser considerados inmediatamente finalizada la intervención, sino que también en el largo plazo (Spittle et al., 2010). En los estudios incluidos en esta revisión, la mayor parte de las evaluaciones finales para medir el resultado de la intervención, se realizaron antes de que el niño cumpliera 24 meses. Esto significa que hay una falta de información sobre el efecto que estos programas tendrían en la edad preescolar y escolar del niño. Futuras investigaciones debiesen medir resultados a largo plazo, teniendo en cuenta el impacto potencial de las intervenciones sobre la participación del niño en las actividades de la vida diaria en edades mayores.

Una preocupación metodológica de larga data en rehabilitación es la falta de información para replicar de manera fidedigna los programas de intervención que han sido exitosos previamente (Kunz et al., 2006; Whyte \& Hart, 2003). La fidelidad de tratamiento se refiere al grado de exactitud con que éste es proporcionado en relación a lo que se describe en el protocolo de intervención. Por ende, la generación de protocolos con información detallada sobre cada componente del tratamiento garantizaría la réplica más exacta de los programas existentes y permitiría una mejor identificación de los componentes terapéuticos que conducen a mayores resultados en el desarrollo motor infantil (Kunz et al., 2006). Cabe agregar que ningún estudio en esta revisión detalla de qué manera fue evaluada y garantizada la fidelidad de los programas implementados.

Por último, a pesar que los ECAs se consideran el mejor diseño metodológico para establecer la efectividad de los PIT, éstos no describen los procesos y mecanismos que conducen a los resultados obtenidos (Watson et al., 2004). En consecuencia, esto podría representar un desafío para aislar y reconocer las caracte- 
rísticas terapéuticas que impulsan la eficacia de algunas intervenciones y el fracaso de otras (Campbell et al., 2000). Algunos autores han señalado que la combinación de una evaluación de impacto con una evaluación de proceso podría ser útil para una comprensión más amplia de los resultados (Bonell et al., 2012; Craig et al., 2008). De acuerdo con esta idea, la evaluación del impacto permitía evaluar el nivel de éxito que ha tenido la intervención, y la evaluación del proceso entregaría más información acerca de los componentes específicos del tratamiento que se asocian con una mejora en el desarrollo motor y aumentar la posibilidad de replicar las intervenciones más exitosas en otros entornos terapéuticos (Blauw-Hospers et al., 2011).

\section{REFERENCIAS BIBLIOGRÁFICAS}

Aisen, M. L., Kerkovich, D., Mast, J., Mulroy, S., Wren, T. A., Kay, R. M. \& Rethlefsen, S. A. (2011). Cerebral palsy: Clinical care and neurological rehabilitation. The Lancet Neurology, 10(9), 844-852. Doi: 10.1016/s1474-4422(11)70176-4.

Ashwal, S., Russman, B. S., Blasco, P. A., Miller, G., Sandler, A., Shevell, M. \& Stevenson, R. (2004). Practice Parameter: Diagnostic assessment of the child with cerebral palsy: Report of the Quality Standards Subcommittee of the American Academy of Neurology and the Practice Committee of the Child Neurology Society. Neurology, 62(6), 851-863. Doi: 10.1212/01.wnl.0000117981.35364.1b.

Badr, L. K., Garg, M. \& Kamath, M. (2006). Intervention for infants with brain injury: Results of a randomized controlled study. Infant Behavior and Development, 29(1), 80-90.

Baird, G., McConachie, H. \& Scrutton, D. (2000). Parents' perceptions of disclosure of the diagnosis of cerebral palsy. Archives of disease in childhood, 83(6), 475-480.

Bax, M., Goldstein, M., Rosenbaum, P., Leviton, A., Paneth, N., Dan, B. (...) \& Damiano, D. (2005). Proposed definition and classification of cerebral palsy, April 2005. Developmental Medicine \& Child Neurology, 47(08), 571-576.

Beaino, G., Khoshnood, B., Kaminski, M., Pierrat, V., Marret, S., Matis, \& Zupan-Simunek, V. (2010). Predictors of cerebral palsy in very preterm infants: the EPIPAGE prospective population-based cohort study. Developmental Medicine \& Child Neurology, 52(6), 119-125.

Benzies, K. M., Magill-Evans, J. E., Hayden, K. A. \& Ballantyne, M. (2013). Key components of early intervention programs for preterm infants and their parents: a systematic review and meta-analysis. BMC pregnancy and childbirth, 13(1), 1.

Bischof, H. J. (2007). Behavioral and neuronal aspects of developmental sensitive periods. Neuroreport, 18(5), 461-465.
Blauw-Hospers, C. H., \& Hadders-Algra, M. (2005). A systematic review of the effects of early intervention on motor development. Developmental medicine \& child neurology, 47(06), 421-432.

Bonell, C., Fletcher, A., Morton, M., Lorenc, T. \& Moore, L. (2012). Realist randomised controlled trials: a new approach to evaluating complex public health interventions. Social science \& medicine, 75(12), 2299-2306.

Bosanquet, M., Copeland, L., Ware, R. \& Boyd, R. (2013). A systematic review of tests to predict cerebral palsy in young children. Developmental Medicine \& Child Neurology, 55(5), 418-426.

Burger, M. \& Louw, Q. A. (2009). The predictive validity of general movements-a systematic review. European journal of paediatric neurology,13(5), 408-420.

Cameron, E. C., Maehle, V. \& Reid, J. (2005). The effects of an early physical therapy intervention for very preterm, very low birth weight infants: a randomized controlled clinical trial. Pediatric Physical Therapy, 17(2), 107-119.

Campbell, M., Fitzpatrick, R., Haines, A. \& Kinmonth, A. L. (2000). Framework for design and evaluation of complex interventions to improve health. British medical journal, 321(7262), 694-696.

Craig, P., Dieppe, P., Macintyre, S., Michie, S., Nazareth, I. \& Petticrew, M. (2008). Developing and evaluating complex interventions: the new Medical Research Council guidance. British Medical Journal, 337(7676), 979-983.

De Vries, L. S., van Haastert, I. C., Benders, M. J. \& Groenendaal, F. (2011, October). Myth: cerebral palsy cannot be predicted by neonatal brain imaging. In Seminars in fetal \& neonatal medicine, 16(5), 279-287.

Dirks, T. \& Hadders-Algra, M. (2011). The role of the family in intervention of infants at high risk of cerebral palsy: a systematic analysis. Developmental medicine and child neurology, 53, 62-67

Donald, K. A., Kakooza, A. M., Wammanda, R. D., Mallewa, M., Samia, P., Babakir, H. (...) \& Chugani, H. (2014). Pediatric Cerebral Palsy in Africa: Where Are We? Journal of Child Neurology, 30(8), 963-971.

De Graaf-Peters, V. B. \& Hadders-Algra, M. (2006). Ontogeny of the human central nervous system: what is happening when? Early human development, 82(4), 257-266.

Fernández, J., Rego, F., Gómez Conesa, A. \& Pérez López, J. (2012). Efficacy of early physiotherapy intervention in preterm infant motor development - A systematic review —. Journal of Physical Therapy Science, 24(9), 933-940. Doi: 10.1589/jpts.24.933.

Hadders-Algra, M. (2014). Early Diagnosis and Early Intervention in Cerebral Palsy. Frontiers in Neurology, 5, 1-13

Hadders-Algra, M. (2011). Challenges and limitations in early intervention. Developmental Medicine \& Child Neurology, 53(s4), 52-55.

Hadders-Algra, M. (2001). Early brain damage and the development of motor behavior in children: clues for therapeutic intervention? Neural plasticity, 8(1-2), 31-49. 
Hadders-Algra, M. (2004). General movements: a window for early identification of children at high risk for developmental disorders. The Journal of pediatrics, 145(2), S12-S18.

Hadders-Algra, M. (2002). Two distinct forms of minor neurological dysfunction: perspectives emerging from a review of data of the Groningen Perinatal Project. Developmental Medicine \& Child Neurology, 44(8), 561-571.

Hensch, T. K. (2004). Critical period regulation. Annu. Rev. Neurosci, 27, 549-579.

Herskind, A., Greisen, G. \& Nielsen, J. B. (2014). Early identification and intervention in cerebral palsy. Developmental Medicine \& Child Neurology, 57(1), 29-36.

Herskind, A., Greisen, G. \& Nielsen, J. B. (2015). Early identification and intervention in cerebral palsy. Developmental Medicine \& Child Neurology, 57(1), 29-36.

Hielkema, T., Blau-Hospers, C. H., Dirks, T., Drijver-Messelink, M.E., Bos, A. F. \& Hadders-Algra, M. (2011). Does physiotherapeutic intervention affect motor outcome in high-risk infants? An approach combining a randomized controlled trial and process evaluation. Developmental Medicine \& Child Neurology, 53(3), 8-15.

Hielkema, T., Hamer, E. G., Reinders-Messelink, H. A., Maathuis, C. G., Bos, A. F., Dirks, T. (...) \& Hadders-Algra, M. (2010). LEARN 2 MOVE 0-2 years: effects of a new intervention program in infants at very high risk for cerebral palsy; a randomized controlled trial. BMC pediatrics, 10(1), 1.

Himpens, E., Van den Broeck, C., Oostra, A., Calders, P. \& Vanhaesebrouck, P. (2008). Prevalence, type, distribution, and severity of cerebral palsy in relation to gestational age: a meta-analytic review. Developmental Medicine \& Child Neurology, 50(5), 334-340.

Holt, R. L. \& Mikati, M. A. (2011). Care for child development: basic science rationale and effects of interventions. Pediatric neurology, 44(4), 239-253.

Hur, J. J. (1995). Review of research on therapeutic interventions for children with cerebral palsy. Acta Neurologica Scandinavica, 91(6), 423-432.

Jones, M. W., Morgan, E., Shelton, J. E. \& Thorogood, C. (2007). Cerebral palsy: introduction and diagnosis (part I). Journal of Pediatric Health Care, 21(3), 146-152.

Ketelaar, M., Vermeer, A. \& Helders, P. J. (1998). Functional motor abilities of children with cerebral palsy: a systematic literature review of assessment measures. Clinical rehabilitation, 12(5), 369-380.

Knudsen, E. I. (2004). Sensitive periods in the development of the brain and behavior. Journal of cognitive neuroscience, 16(8), 1412-1425.

Kolb, B., Mychasiuk, R., Muhammad, A. \& Gibb, R. (2013). Chapter 2 - brain plasticity in the developing brain. Progress in Brain Research, 207, 35-64. Doi: http://dx.doi.org/10.1016/B978-0-44463327-9.00005-9
Kolb, B., Mychasiuk, R., Williams, P. \& Gibb, R. (2011). Brain plasticity and recovery from early cortical injury. Developmental Medicine \& Child Neurology, 53(s4), 4-8.

Kolb, B., Brown, R., Witt-Lajeunesse, A. \& Gibb, R. (2001). Neural compensations after lesion of the cerebral cortex. Neural plasticity, 8(12), 1-16.

Koldewijn, K., Wolf, M. J., van Wassenaer, A., Meijssen, D., van Sonderen, L., van Baar, A. (...) \& Kok, J. (2009). The Infant Behavioral Assessment and Intervention Program for very low birth weight infants at 6 months corrected age. The Journal of pediatrics, 154(1), 33-38.

Krägeloh-Mann, I. \& Horber, V. (2007). The role of magnetic resonance imaging in elucidating the pathogenesis of cerebral palsy: a systematic review. Developmental Medicine \& Child Neurology, 49(2), 144-151.

Kunz, R., Autti-Rämö, I., Anttila, H., Malmivaara, A. \& Mäkelä, M. (2006). A systematic review finds that methodological quality is better than its reputation but can be improved in physiotherapy trials in childhood cerebral palsy. Journal of clinical epidemiology, 59(12), 1239-1248.

Maher, C. G., Sherrington, C., Herbert, R. D., Moseley, A. M. \& Elkins, M. (2003). Reliability of the PEDro scale for rating quality of randomized controlled trials. Physical therapy, 83(8), 713-721.

Majnemer, A. (1998, March). Benefits of Early Intervention for Children With Developmental Disabilities. In Seminars in Pediatric Neurology 5(1), 62-69.

McIntyre, S., Taitz, D., Keogh, J., Goldsmith, S., Badawi, N. \& Blair, E. V. E. (2013). A systematic review of risk factors for cerebral palsy in children born at term in developed countries. Developmental Medicine \& Child Neurology, 55(6), 499-508.

McIntyre, S., Morgan, C., Walker, K. \& Novak, I. (2011). Cerebral palsydon't delay. Developmental disabilities research reviews, 17(2), 114-129.

Nelson, M. N., White-Traut, R. C., Vasan, U., Silvestri, J., Comiskey, E., Meleedy-Rey, P. (...) \& Patel, M. (2001). One-year outcome of auditory-tactile-visual-vestibular intervention in the neonatal intensive care unit: effects of severe prematurity and central nervous system injury. Journal of Child Neurology, 16(7), 493-498.

Nimchinsky, E. A., Sabatini, B. L. \& Svoboda, K. (2002). Structure and function of dendritic spines. Annual review of physiology, 64(1), 313-353.

Odding, E., Roebroeck, M. E. \& Stam, H. J. (2006). The epidemiology of cerebral palsy: Incidence, impairments and risk factors. Disability and Rehabilitation, 28(4), 183-191. Doi: 10.1080/09638280500158422. 
Ohgi, S., Fukuda, M., Akiyama, T. \& Gima, H. (2004). Effect of an early intervention programme on low birthweight infants with cerebral injuries. Journal of Paediatrics and Child Health, 40(12), 689-695. Doi: 10.1111/j.1440-1754.2004.00512.x.

Siebes, R. C., Wijnroks, L. \& Vermeer, A. (2002). Qualitative analysis of therapeutic motor intervention programmes for children with cerebral palsy: An update. Dev Med Child Neurol Developmental Medicine \& Child Neurology, 44(09). Doi: 10.1017/ s0012162201002638.

Simeonsson, R., Leonardi, M., Lollar, D., Bjorck-Akesson, E., Hollenweger, J. \& Martinuzzi, A. (2003). Applying the International Classification of Functioning, Disability and Health (ICF) to measure childhood disability. Disability and Rehabilitation, 25(11-12), 602610. Doi: 10.1080/0963828031000137117.

Skiöld, B., Eriksson, C., Eliasson, A., Ådén, U. \& Vollmer, B. (2013). General movements and magnetic resonance imaging in the prediction of neuromotor outcome in children born extremely preterm. Early Human Development, 89(7), 467-472. Doi: 10.1016/j.earlhumdev.2013.03.014.

Spittle, A., Orton, J., Anderson, P., Boyd, R. \& Doyle, L. W. (2012). Early developmental intervention programmes post-hospital discharge to prevent motor and cognitive impairments in preterm infants. $\mathrm{Co}$ chrane Database of Systematic Reviews. Doi: 10.1002/14651858. cd005495.pub3.

Spittle, A. J., Anderson, P. J., Lee, K. J., Ferretti, C., Eeles, A., Orton, J. (...) \& Doyle, L. W. (2010). Preventive Care at Home for Very Preterm
Infants Improves Infant and Caregiver Outcomes at 2 Years. Pediatrics, 126(1). Doi: 10.1542/peds.2009-3137.

Van den Bergh, B. R., Mulder, E. J., Mennes, M. \& Glover, V. (2005). Antenatal maternal anxiety and stress and the neurobehavioural development of the fetus and child: links and possible mechanisms. A review. Neuroscience \& Biobehavioral Reviews, 29(2), 237-258.

Verhagen, A. P., Vet, H. C., Bie, R. A., Kessels, A. G., Boers, M., Bouter, L. M. \& Knipschild, P. G. (1998). The Delphi List: A criteria for quality assessment of randomized clinical trials for conductiong systematic reviews developed by Delphi consensus. Journal of Clinical Epidemiology, 51(12), 1235-1241. Doi: 10.1016/s0895-4356(98)00131-0 .

Watson, B., Procter, S. \& Cochrane, W. (2004). Using randomised controlled trials (RCTs) to test service interventions: Issues of standardisation, selection and generalisability. Nurse Researcher, 11(3), 2842. Doi: 10.7748/nr2004.04.11.3.28.c6203.

Weindling, A. (1996). A randomized controlled trial of early physiotherapy for high-risk infants. Acta Pediatrica, 204(3), 283-299.

Weinstock, M. (2008). The long-term behavioural consequences of prenatal stress. Neuroscience and Biobehavioral Reviews, 32(6), 10731086.

Zigler, E. F. (2000). Handbook of early childhood intervention. J. P. Shonkoff \& S. J. Meisels (Eds.). Cambridge University Press. 